\title{
Role of routine upper endoscopy before bariatric surgery in the Middle East population: a review of 1278 patients
}

\section{(잉 $\odot$}

\author{
Authors \\ Mazrouei ${ }^{1}$, Faisal Badri ${ }^{1}$ \\ Institutions \\ 1 General Surgery Department, Rashid Hospital, DHA, \\ Dubai, United Arab Emirates \\ 2 Gastroenterology Department, Rashid Hospital, DHA, \\ Dubai, United Arab Emirates
}

Bassem Abou Hussein ${ }^{1}$, Ali Khammas ${ }^{1}$, Mariam Shokr ${ }^{1}$, Maiyasa Majid ${ }^{1}$, Mariam Sandal' ${ }^{1}$, Sameer Al Awadhi ${ }^{2}$, Alya Al

submitted 30.1 .2018

accepted after revision 11.6 .2018

Bibliography

DOI https://doi.org/10.1055/a-0659-2395 |

Endoscopy International Open 2018; 06: E1171-E1176

(c) Georg Thieme Verlag KG Stuttgart · New York

ISSN 2364-3722

Corresponding author

Bassem Mahmoud Abou Hussein, General Surgery

Department, Rashid Hospital, Oud Meitha Street, Dubai,

PO Box: 4545, United Arab Emirates

Fax: +971-4-3113222

bassem.abouhussein@gmail.com

\section{ABSTRACT}

Introduction The routine use of preoperative endoscopy for patients undergoing bariatric surgery is controversial. Although many surgeons find it unnecessary, others still consider it a mandatory tool in preoperative assessment.
Patients and methods We reviewed the medical records of all patients who had undergone preoperative endoscopy before bariatric surgery in Rashid Hospital between January 2013 and June 2016. The patients were divided into three groups: Group 0 included patients with normal endoscopy; Group 1 included patients with abnormalities that did not affect the timing or type of procedure; Group 2 included patients with abnormalities that had a direct impact on the procedure.

Results The files of 1473 patients were reviewed. Endoscopy results were not present in 195 files, so those patients were excluded, and the remaining 1278 files were included. The mean age of patients was $41.3 \pm 12.7$ years, $61 \%$ were female and $39 \%$ were male. The mean body mass index (BMI) was $43.7 \pm 8 \mathrm{~kg} / \mathrm{m}^{2}$. Endoscopy was normal in $10.6 \%$ of patients and abnormal in $89.4 \%$. The most common abnormalities were gastritis, positive Campylobacter-like organism test (CLO test), gastroesophageal reflux disease (GERD) with esophagitis and hiatal hernia. Group 0 included $10.6 \%$ of patients ( $n=135$ ), Group 1 included $25.6 \%$ of patients $(n=327)$, while Group 2 included $63.8 \%$ of patients $(n=816)$.

Conclusion Routine endoscopy seems to play an important part in the preoperative preparation of patients planned for bariatric surgery in the Middle East population. Further studies or meta-analysis could help in building up clear solid evidence and guidelines that could be approved by international bariatric associations with regard to indications for preoperative upper endoscopy in bariatric patients.

\section{Introduction}

Morbid obesity has been associated with a number of gastrointestinal diseases. It has been shown to be an important risk factor in the development of gastroesophageal reflux disease (GERD), erosive esophagitis, and hiatal hernia [1]. Upper digestive diseases are $2-3$ times more common in obese than in normal weight individuals, including erosive esophagitis, gastroesophageal reflux, hiatal hernia, Barrett's esophagus, and Helicobacter pylori (H. pylori) infection [2].
The possible benefits of using preoperative upper endoscopy as a tool in the assessment of obese patients before bariatric surgery have not yet been determined. Some physicians consider it mandatory to rule out any upper gastrointestinal disease that may affect surgery because it would be difficult to assess all parts of the gastrointestinal tract after some bariatric procedures; however, others still believe that endoscopy is unnecessary and may expose patients to an unnecessary procedure, sedation, and possible complications, especially since most obese patients have multiple comorbidities. 
- Table 1 Endoscopic findings among patients in the study.

\begin{tabular}{|c|c|c|c|}
\hline Group & Classification of findings & Findings & No. of patients \\
\hline Group 0 & No findings & Normal endoscopy & 135 \\
\hline \multirow[t]{4}{*}{ Group 1} & Non-significant findings & Simple gastritis & 419 \\
\hline & & Simple duodenitis & 127 \\
\hline & & Positive CLO test with no other significant findings & 364 \\
\hline & & Small hiatal hernia $<2 \mathrm{~cm}$ & 115 \\
\hline \multirow[t]{7}{*}{ Group 2} & Significant findings & Esophagitis/GERD & 259 \\
\hline & & Barrett's esophagus & 38 \\
\hline & & Large hiatal hernia > $2 \mathrm{~cm}$ & 189 \\
\hline & & Erosive gastritis/duodenitis & 241 \\
\hline & & Positive CLO test with other significant findings & 215 \\
\hline & & Peptic ulcer & 85 \\
\hline & & Mass lesion/cancer & 4 \\
\hline
\end{tabular}

Many authors suggest performing esophagogastroduodenoscopy (EGD) in all patients before bariatric procedures because of the lack of correlation between patient symptoms and EGD findings [3]. On the other hand, many other investigators advocate a selective approach for asymptomatic patients because of the relatively weak clinical relevance of the majority of lesions discovered on routine EGD along with the cost and invasiveness of EGD [3].

\section{Patients and methods}

All patients presenting to our department requesting weight reduction surgery were reviewed by our bariatric team. Bariatric surgery was offered to patients who fulfilled the criteria of our hospital bariatric unit protocol and were selected by the bariatric team as candidates for surgery (patients with body mass index $(\mathrm{BMI})>35 \mathrm{~kg} / \mathrm{m}^{2}$ with known comorbidities, or patients with $\mathrm{BMI}>40 \mathrm{~kg} / \mathrm{m}^{2}$ without known comorbidities). The type of procedure was discussed and agreed between the patient and the bariatric team after reviewing all investigations.

Gastroscopy is routinely performed in our hospital before any bariatric procedure in order to assess the upper gastrointestinal tract for any abnormal findings. Gastroscopy was performed by experienced endoscopists in our gastroenterology department under local spray anesthesia with or without sedation and the findings were recorded in a hospital database system. The Campylobacter-like organism test (CLO test) to detect $H$. pylori was carried out in all endoscopic procedures and, in the presence of any ulcer or lesion, multiple biopsies were taken. We retrospectively reviewed the medical records of all patients admitted for bariatric surgery in our center between January 2013 and June 2016. The study included only asymptomatic patients. Patients who were symptomatic, those taking NSAIDs or steroids, and those with a previous history of gastric diseases were not included. In total, 1473 files were reviewed; however, gastroscopy results were not available in 195 files (gastroscopy done in other institutes) and these files were excluded from the study; the remaining 1278 patients were included in data collection.

Data collected included age, sex, preoperative BMI, all gastroscopy findings, duration of endoscopy, and any associated complications.

Patients were divided into three groups according to the gastroscopy findings: Group 0 included patients with normal endoscopy; Group 1 included patients with endoscopic findings that were not significant and did not interfere with the procedure plan; Group 2 included patients with significant findings that affected the procedure in some way (procedure postponed or cancelled, or the type of procedure was changed). In the presence of multiple findings on gastroscopy, the most serious abnormality with the most significant impact on the bariatric procedure was considered for counting and data analysis.

\section{Results}

Between January 2013 and June 2016, 1473 patients were admitted to our unit for bariatric surgery, and data from the 1278 files available were collected and analyzed. The mean age of the patients was $41.3 \pm 12.7$ years, $61 \%$ were female and $39 \%$ were male, and the mean BMI was $43.7 \pm 8 \mathrm{~kg} / \mathrm{m}^{2}$.

Endoscopic findings were variable among different patients ( $>$ Table 1, $>$ Fig. 1). Normal endoscopy with no findings was present in 135 patients (10.6\%) while a single or multiple abnormal findings were present in 1143 patients (89.4\%). A single finding was present in $316 / 1278$ patients (24.7\%), while multiple findings were present in $827 / 1278$ patients (64.7\%). Among the non-significant findings, simple gastritis (erythema of the gastric mucosa with no erosions or bleeding) was present in 


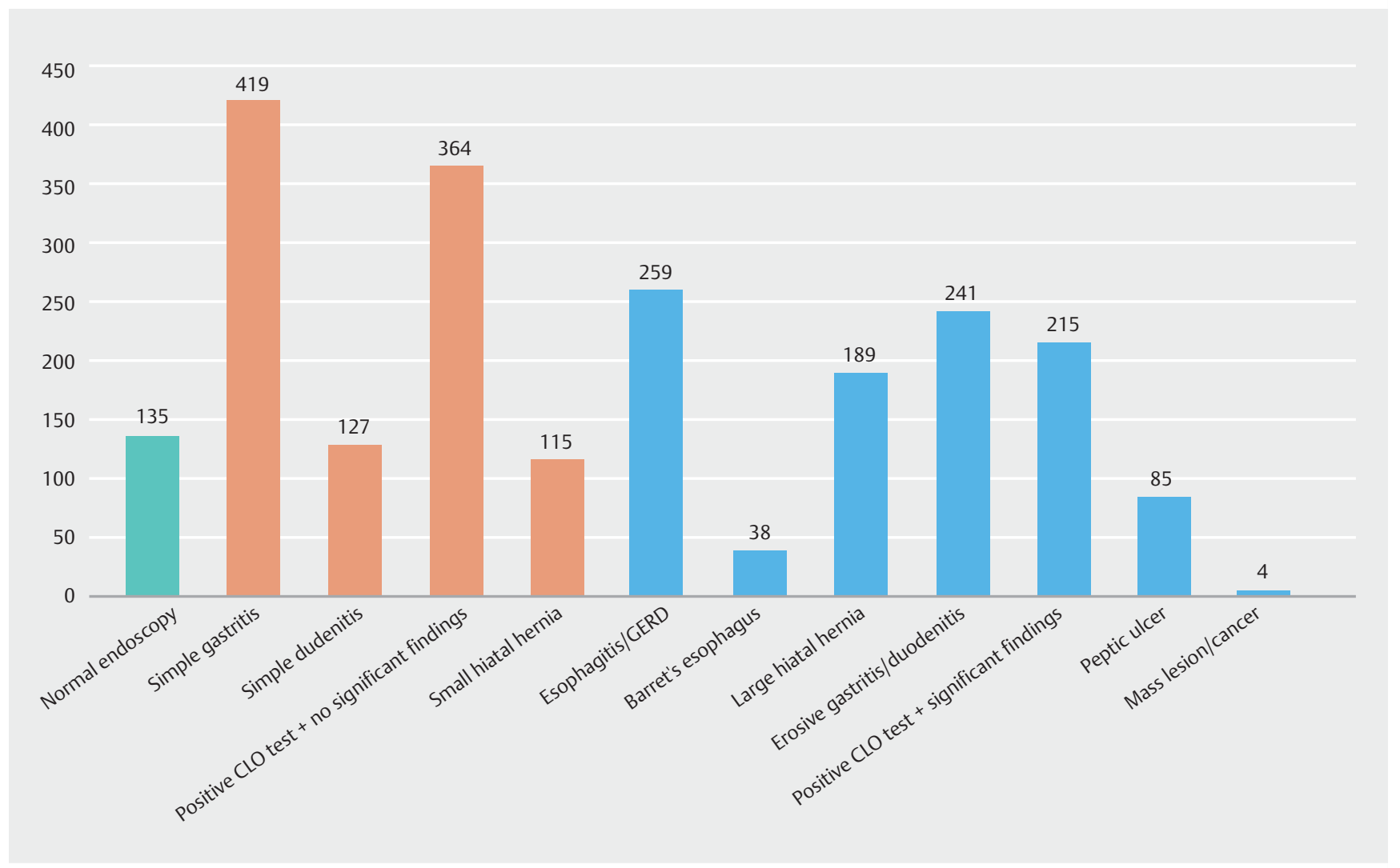

- Fig. 1 Distribution of endoscopic findings among patients in the study.

$419 / 1278$ patients $(32.8 \%$ ) while simple duodenitis was found in $127 / 1278$ patients $(9.9 \%)$, and a small hiatal hernia $(<2 \mathrm{~cm})$ was present in $115 / 1278$ patients $(9.0 \%)$. On the other hand, significant findings were more common and included: esophagitis and GERD in 259/1278 patients (20.3\%), Barrett's esophagus in $38 / 1278$ patients $(3.0 \%)$, large hiatal hernia $(>2 \mathrm{~cm})$ in $189 / 1278$ patients (14.8\%), erosive gastritis (gastric mucosal damage or erosions that could bleed easily) and duodenitis in $241 / 1278$ patients (18.9\%), positive CLO test in 579/1278 patients $(45.3 \%)$, peptic ulcer in $85 / 1278$ patients $(6.7 \%)$, and a mass lesion or cancer in $4 / 1278$ patients $(0.3 \%)$. A positive CLO test was associated with other significant findings in 215 patients, while 364 patients had a positive CLO test with no other associated significant findings.

Patients with normal endoscopy proceeded directly to bariatric surgery. Patients with simple gastritis and duodenitis were started on oral proton pump inhibitors and diet modification and then proceeded to surgery. Patients with small hiatal hernias proceeded directly to surgery if there were no symptoms, and the hernia was assessed intraoperatively. Patients with severe esophagitis, GERD, Barrett's esophagus or large hiatal hernias were started on diet modification and proton pump inhibitors but these conditions were considered to be an indication for gastric bypass and a contraindication for sleeve gastrectomy. In this study, we did not consider the grading of GERD as an indicator for diversion surgery; however, we considered GERD to be severe and a contraindication for sleeve gastrect- omy once it was associated with esophagitis and/or Barrett's esophagus.

For patients with a positive CLO test and no other significant findings, bariatric surgery was postponed (for approximately 6 weeks) until they had received a full course of treatment (triple therapy as advised by the gastroenterology team), but these patients were not counted in the significant group because many authors $[4,5]$ do not consider a positive CLO test alone to be a significant finding before bariatric surgery; these patients had a urea breath test to ensure eradication of $\mathrm{H}$. pylori after completion of treatment in addition to clinical assessment and then surgery was performed. The procedures for patients with a positive CLO test and other significant findings such as erosive gastritis/duodenitis or peptic ulcers (duodenal/gastric) were postponed until the patients had received full treatment. If repeat upper endoscopy and CLO test after 8-10 weeks showed resolution of erosions or healed ulcers and eradication of $H$. pylori, they then proceeded to surgery.

All patients were kept on daily proton pump inhibitors for 36 months after bariatric surgery. The procedure was completely cancelled for four patients in whom mass lesions/cancer was detected and they were treated accordingly; one patient had a lower esophageal cancer, one patient had gastric adenocarcinoma and two patients had gastrointestinal stromal tumors (GIST). No complications were reported during or after endoscopy among all patients in this series. 


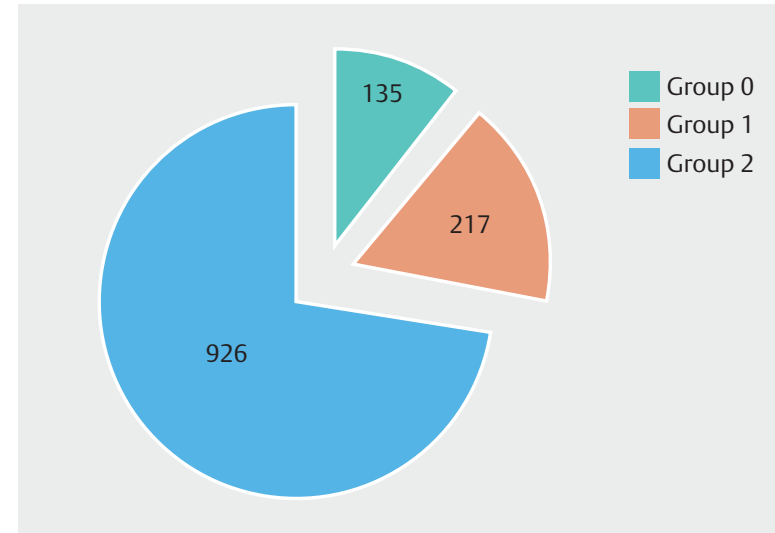

Fig. 2 Distribution of patients in groups according to clinical significance of endoscopic findings.

The patients were assigned to the groups as previously discussed: Group 0 with normal endoscopy included $10.6 \%$ of patients $(n=135)$, Group 1 with non-significant findings alone that did not affect the timing or type of planned surgery included $25.6 \%$ of patients $(n=327)$, while the majority of patients (63.8\%, $n=816$ ) were assigned to Group 2 with significant findings that necessitated delaying the procedure (for approximately $10 \pm 2$ weeks), changing the type of procedure or even canceling it ( $\mathbf{F i g} \mathbf{2}$ ).

Since 2013, the bariatric surgical protocol at our hospital has followed this classification. For all patients in Group 2, bariatric surgery was postponed and they were operated later after finishing their treatment. For those patients who needed to change the type of procedure, the reason was explained to them and they agreed on the change. For those patients who had pathologies necessitating canceling the procedure, they were referred to the appropriate facility to continue work-up and treatment of the new pathology detected.

\section{Discussion}

The implementation of routine upper endoscopy in the preoperative preparation of all obese patients presenting for bariatric surgery remains one of the most controversial topics among surgeons and different bariatric units. Patients who are planned for restrictive procedures such as a sleeve gastrectomy or an adjustable gastric banding may be at greater risk of worsening of gastroesophageal reflux, Barrett's esophagus, and other complications $[6,7]$. These patients will need better counseling and the selection of a more appropriate bariatric procedure. Moreover, bypass procedures such as Roux-en- $Y$ gastric bypass will result in an inaccessible foregut which will make future evaluation of the upper gastrointestinal tract problematic, especially if these patients have an increased risk or pre-existing gastroduodenal pathology [8]. On the other hand, many other investigators advocate a selective approach for asymptomatic patients because of the relatively weak clinical relevance of the majority of lesions discovered on routine EGD along with the cost and invasiveness of the $\operatorname{EGD}[9,10]$. Despite the presence of other modalities for evaluating the gastrointestinal tract such as barium meal, upper endoscopy remains by far the procedure of choice.

Guidelines pertaining to preoperative endoscopic screening are uncertain. The American Society for Gastrointestinal Endoscopy [11] does not recommend blanket screening but suggests the decision should be individualized to the patient. The European Association for Endoscopic Surgery [12], however, recommends preoperative endoscopy but provides scarce supporting evidence. To date, the American Society for Metabolic and Bariatric Surgery recommends endoscopic evaluation if clinically indicated [13], but there are no recommendations from the International Federation for the Surgery of Obesity, or any Asian consensus on this topic.

The prevalence of abnormal EGD findings in morbidly obese patients ranged from $30 \%$ to $89.7 \%$ [1]. The most commonly reported abnormalities were gastritis (13.6-28.7\%), hiatal hernias $(9-40 \%)$, and esophagitis (9.2-17\%). A great variation is also noted in the prevalence of clinically significant EGD abnormalities, with rates ranging from $9.4 \%$ to $61.5 \%$. Lee et al. demonstrated an overall prevalence of abnormal EGD of $51.1 \%$, with $27.2 \%$ classified as clinically significant [1].

Frigg et al. advocated routine gastroscopy before bariatric surgery because of the high prevalence of upper gastrointestinal lesions [14]. Sharaf and colleagues further documented clinically important findings in $61.5 \%$ of patients having routine preoperative gastroscopy [15]. In a large series of 626 patients reported by Muñoz and colleagues [16], abnormalities were found in $46 \%$ of patients including early gastric cancer. On the other hand, other studies found that significant abnormalities affecting the procedure were not common. Schigt et al. [17] and Loewen et al. [9] found that gastroscopic findings only delayed or changed surgical management in $1.3 \%$ and $<1 \%$ of cases respectively. A large systematic review and meta-analysis by Bennett and colleagues [18] concluded that preoperative endoscopy in average risk, asymptomatic bariatric surgery patients should be considered optional, as the proportion of endoscopies that resulted in important changes in management was low.

An explanation for the extremely variable results of significant findings among different studies is that different surgeons deal with endoscopic findings in different levels of significance with no clear definition of which findings are clinically significant and directly affect the planned surgery. This has led to different protocols in the management of gastroscopic findings $[19,20]$. Some surgeons believe that conditions such as hiatal hernia and gastroesophageal reflux are natural consequences of obesity and they will disappear naturally with weight reduction after a successful bariatric surgery. Others believe that these conditions are vital aspects of obesity management, which have significant implications for the selection of subsequent surgical procedures. In their study, $\mathrm{Ng}$ et al. [21] considered erosive gastritis or esophagitis, benign or malignant tumors and ulcer disease to be clinically significant and they correlated hiatal hernia with symptoms and explored it intraoperatively to decide about crural repair. They found that crural repair was deemed to be sufficient and none of the patients had 
- Table 2 Comparison of our results with results of previous similar studies.

\begin{tabular}{|c|c|c|c|c|c|c|}
\hline Reference, year & $\begin{array}{l}\text { Number of } \\
\text { patients }\end{array}$ & $\begin{array}{l}\text { Mean age } \\
\text { (years) }\end{array}$ & $\begin{array}{l}\text { Mean BMI } \\
\left(\mathrm{kg} / \mathrm{m}^{2}\right)\end{array}$ & $\begin{array}{l}\text { \% Normal } \\
\text { endoscopy }\end{array}$ & $\begin{array}{l}\text { \% Abnormal } \\
\text { endoscopy }\end{array}$ & $\begin{array}{l}\text { \% Significant } \\
\text { abnormalities }\end{array}$ \\
\hline Sharaf et al. [15], 2004 & 195 & 41.2 & 48.9 & 10.3 & 89.7 & 61.5 \\
\hline Zeni et al. [22], 2006 & 159 & 41.1 & 49.7 & 33.4 & 66.6 & 9.4 \\
\hline Muñoz et al. [16], 2008 & 626 & 38.5 & 42 & 54 & 46 & - \\
\hline Loewen et al. [9], 2008 & 447 & 40.6 & 48.6 & 70 & 30 & 18 \\
\hline Schigt et al. [17], 2014 & 523 & 44.3 & 46.6 & 49.9 & 50.1 & 14.7 \\
\hline Lee et al. [1], 2017 & 268 & 39.1 & 40.3 & 48.9 & 51.1 & 27.2 \\
\hline This study & 1473 & 41.3 & 43.7 & 10.6 & 89.4 & 63.8 \\
\hline
\end{tabular}

a modification of surgery to a gastric bypass. In our unit, large symptomatic hiatal hernias are repaired; esophagitis and Barrett's esophagus are considered to be a contraindication for sleeve gastrectomy; erosive gastritis, duodenitis and peptic ulcers are treated before proceeding to surgery. Cancer and GIST were detected in four patients and were considered to be an absolute contraindication for bariatric surgery and these patients were treated according to their findings.

We compared our results with other previous studies $>$ (Table 2); there are some common results, especially those with significant abnormalities detected, although some other studies did not show similar findings. To our knowledge, and after a thorough review of Pubmed, Medscape, and Elsevier, our study represents the largest single-center study reviewing upper endoscopy results before bariatric surgery published in the English language literature. These significant numbers suggest that preoperative upper endoscopy is indicated and may need to be implemented in all bariatric centers.

\section{Conclusion}

Despite the fact that some surgeons are still reluctant to perform routine upper endoscopy before bariatric procedures; we found high clinical significance in doing so. The procedure was canceled, changed or postponed in $63.8 \%$ of patients due to significant abnormal findings in gastroscopy. There were no complications (morbidity or mortality) in the 1278 patients reviewed. The authors suggest that further studies with prospective follow-up could help to build more solid evidence and consequently help to implement upper endoscopy routinely before bariatric surgery.

\section{Competing interests}

None

\section{References}

[1] Lee ], Wong SKH, Liu SYW et al. Is preoperative upper gastrointestinal endoscopy in obese patients undergoing bariatric surgery mandatory? An Asian perspective Obes Surg 2017; 27: 44 - 50

[2] Clinical Guidelines on the Identification, Evaluation, and Treatment of Overweight and Obesity in Adults - The Evidence Report. National Institutes of Health. Obes Res 1998; 6: (Suppl. 02): 51S-209S

[3] Abd Ellatif ME, Alfalah H, Asker WA et al. Place of upper endoscopy before and after bariatric surgery: A multicenter experience with 3219 patients. World J Gastrointest Endosc 2016; 8: 409-417

[4] Almazeedi S, Al-Sabah S, Alshammari D et al. The impact of Helicobacter pylori on the complications of laparoscopic sleeve gastrectomy. Obes Surg 2014; 24: 412-415

[5] Carabotti M, D'Ercole C, lossa A et al. Helicobacter pylori infection in obesity and its clinical outcome after bariatric surgery. World J Gastroenterol 2014; 20: 647-653

[6] Merrouche M, Sabaté JM, Jouet P et al. Gastro-esophageal reflux and esophageal motility disorders in morbidly obese patients before and after bariatric surgery. Obes Surg 2007; 17: 894 - 900

[7] Greenstein RJ, Nissan A, Jaffin B. Esophageal anatomy and function in laparoscopic gastric restrictive bariatric surgery: implications for patient selection. Obes Surg 1998; 8: 199-206

[8] Bernante P, Breda C, Zangrandi F et al. Emergency sleeve gastrectomy as rescue treatment for acute gastric necrosis due to type II paraesophageal hernia in an obese woman with gastric banding. Obes Surg 2008; 18: $737-741$

[9] Loewen M, Giovanni ], Barba C. Screening endoscopy before bariatric surgery: a series of 448 patients. Surg Obes Relat Dis 2008; 4: 709 712

[10] Peromaa-Haavisto P, Victorzon M. Is routine preoperative upper GI endoscopy needed prior to gastric bypass? Obes Surg 2013; 23: 736 739

[11] ASGE Standards of Practice Committee. The role of endoscopy in the bariatric surgery patient. Gastrointest Endosc 2015; 81: 1063 - 1072

[12] Sauerland S, Angrisani L, Belachew M et al. Obesity surgery: evidencebased guidelines of the European Association for Endoscopic Surgery (EAES). Surg Endosc 2005; 19: 200-221

[13] Mechanick JI, Youdim A, Jones DB et al. Clinical practice guidelines for the perioperative nutritional, metabolic, and nonsurgical support of the bariatric surgery patient - 2013 update: cosponsored by American Association of Clinical Endocrinologists, the Obesity Society, and American Society for Metabolic \& Bariatric Surgery. Surg Obes Relat Dis 2013; 9: 159-191 
[14] Frigg A, Peterli R, Zynamon A et al. Radiologic and endoscopic evaluation for laparoscopic adjustable gastric banding: preoperative and follow-up. Obes Surg 2001; 11: 594-599

[15] Sharaf RN, Weinshel EH, Bini EJ et al. Endoscopy plays an important preoperative role in bariatric surgery. Obes Surg 2004; 14: $1367-$ 1372

[16] Muñoz R, Ibáñez L, Salinas J et al. Importance of routine preoperative upper $\mathrm{Gl}$ endoscopy: why all patients should be evaluated? Obes Surg 2009; 19: 427-431

[17] Schigt A, Coblijn U, Lagarde S et al. Is esophagogastroduodenoscopy before Roux-en-Y gastric bypass or sleeve gastrectomy mandatory? Surg Obes Relat Dis 2014; 10: 411 -417; quiz 565-566
[18] Bennett S, Gostimir M, Shorr R et al. The role of routine preoperative upper endoscopy in bariatric surgery: a systematic review and metaanalysis. Surg Obes Relat Dis 2016; 12: 1116 - 1125

[19] Korenkov M, Köhler L, Yücel $N$ et al. Esophageal motility and reflux symptoms before and after bariatric surgery. Obes Surg 2002; 12: $72-76$

[20] Miller K, Hell E. Laparoscopic surgical concepts of morbid obesity. Langenbecks Arch Surg 2003; 388: 375-384

[21] Ng JY, Cheng AKS, Kim G et al. Is elective gastroscopy prior to bariatric surgery in an Asian cohort worthwhile? Obes Surg 2016; 26: 2156 2160

[22] Zeni TM, Frantzides CT, Mahr C et al. Value of preoperative upper endoscopy in patients undergoing laparoscopic gastric bypass. Obes Surg 2006; 16: $142-146$ 